\title{
Interleukin 6 as an energy allocator in muscle tissue
}

\author{
Timothy M. Kistner $\left(\mathbb{1}^{1 凶}\right.$, Bente K. Pedersen $\mathbb{1}^{2 凶}$ and Daniel E. Lieberman ${ }^{1}{ }^{1 凶}$
}

\begin{abstract}
Extensive research has shown that interleukin 6 (IL-6) is a multifunctional molecule that is both proinflammatory and anti-inflammatory, depending on the context. Here, we combine an evolutionary perspective with physiological data to propose that IL-6's context-dependent effects on metabolism reflect its adaptive role for short-term energy allocation. This energy-allocation role is especially salient during physical activity, when skeletal muscle releases large amounts of IL-6. We predict that during bouts of physical activity, myokine IL-6 fulfills the three main characteristics of a short-term energy allocator: it is secreted from muscle in response to an energy deficit, it liberates somatic energy through lipolysis and it enhances muscular energy uptake and transiently downregulates immune function. We then extend this model of energy allocation beyond myokine IL-6 to reinterpret the roles that IL-6 plays in chronic inflammation, as well as during COVID-19-associated hyperinflammation and multiorgan failure.
\end{abstract}

L-6 was originally characterized as a B-cell differentiation factor ${ }^{1}$. Since then, research has recast IL-6 as a pleiotropic cytokine with roles in the acute-phase response, hematopoiesis, maintenance of bone and skeletal muscle mass, central nervous system activity and metabolism. Correspondingly, multiple cell types, including leukocytes, adipocytes and myocytes, are known to secrete IL-6 where it can act in an autocrine, paracrine or endocrine manner ${ }^{2-4}$. IL-6 is present in diverse taxa ${ }^{5}$, but between species the protein is highly variable, with the human amino acid sequence being only $41 \%$ homologous to the murine version ${ }^{6}$.

Molecularly, IL-6 uses three pathways to signal intracellularly (Fig. 1). Classical signalling depends on IL-6 binding to membrane-bound IL-6 receptors (IL-6R) and subsequent activation of transmembrane protein gp130. As IL-6R is expressed only on select cell types, such as hepatocytes, various leukocyte subsets, adipocytes and myocytes ${ }^{7-9}$, this signalling method is limited. Trans-signalling relies on soluble IL-6 receptors (sIL-6R) that are either generated by cleavage of IL-6R by metalloproteases, such as ADAM-10 and ADAM-17 (ref. ${ }^{10}$ ), or through alternative splicing of IL-6R messenger RNA (mRNA) ${ }^{11}$. Once bound to IL-6, the resulting sIL-6R-IL-6 complex interacts with widely expressed gp130 to circumvent the tissue constraints of classical signalling. A variety of soluble gp130 receptors then modulate the degree of trans-signalling by inhibiting the sIL-6R-IL-6 complex to varying degrees ${ }^{12}$. Recently, a third signalling pathway, cluster signalling, was discovered that also circumvents the tissue constraints of classical signalling. In this pathway, transmitting cells present an IL-6R-IL-6 complex to receiving cells, which induces gp130 receptor activation on the receiving cell ${ }^{13}$. Regardless of how the signalling complex is established, gp130 dimerization can activate several intracellular cascades, including phosphatidylinositol 3-kinase (PI3K), AMP-activated protein kinase (AMPK) and Janus kinase (JAK)-STAT (primarily STAT3) pathways ${ }^{10,14}$.

Basal elevation of IL- 6 has long been correlated with disease severity in several diseases, such as rheumatoid arthritis (RA), Crohn's disease and atherosclerosis ${ }^{15}$. In the case of RA, IL-6 is causal to disease pathogenesis, and tocilizumab, a monoclonal antibody against IL-6R, is used to treat people with the disease ${ }^{16}$. These findings led to IL-6 initially being categorized as a proinflammatory cytokine. Now, however, there is a growing consensus that IL-6 also has regenerative and anti-inflammatory functions, especially when secreted by skeletal muscle during physical activity (PA) ${ }^{17}$. IL-6's effects on metabolism are similarly varied. Elevated plasma IL-6 levels increase the likelihood of developing type 2 diabetes ${ }^{18}$ and can cause insulin resistance in several tissues in cell models and rodent experiments ${ }^{19,20}$. Despite these effects, IL- 6 also promotes anti-diabetogenic processes, such as enhancing muscular glucose uptake $^{21}$, stimulating pancreatic insulin secretion ${ }^{22}$ and promoting lipolysis and fat oxidation ${ }^{23}$. Moreover, IL-6 inhibits appetite ${ }^{24}$ and delays gastric emptying, which reduces postprandial glycaemia ${ }^{25}$. Aside from metabolism, IL-6 regulates manifold processes, including skeletal muscle hypertrophy ${ }^{26}$ and bone remodelling ${ }^{27}$. These and other functions (summarized in Table 1) raise the question of why this one cytokine regulates so many different cell types in diverse and sometimes discrepant ways.

While hundreds of studies have elucidated how IL-6 functions in a wide range of contexts, here we integrate metabolic, immunological and physiological data with an evolutionary perspective to attempt to explain why this molecule has such disparate and seemingly contradictory functions. We propose that IL-6 evolved to modulate energy allocation in response to metabolic stress in a range of tissues, thus accounting for its diverse effects in multiple contexts. To explore this evolutionary hypothesis, we review how principles of life-history theory suggest a model of IL-6 as a short-term energy allocator. Next, we apply our model to IL-6 secretion by skeletal muscle during PA, hereafter referred to as myokine IL-6. We then extend our model to physical inactivity and other physiological processes involving IL-6.

\section{Long-term versus short-term energy allocation}

All organisms have finite energy reserves to spend on growth, somatic maintenance, storage, physical activity and reproduction. According to life-history theory, natural selection favours mechanisms that allocate energy towards these functions differentially across the life cycle to maximize reproductive success ${ }^{28}$. As a result, organisms ultimately benefit from investing as much energy as possible towards reproduction. Weeks or months of positive energy balance signal that it is favourable to invest energy in a potential

'Department of Human Evolutionary Biology, Harvard University, Cambridge, MA, USA. ${ }^{2}$ Centre of Inflammation and Metabolism/Centre for Physical Activity Research (CIM/CFAS), Rigshospitalet, University of Copenhagen, Copenhagen, Denmark. $\varpi_{e}$-mail: tkistner@g.harvard.edu; Bente.Klarlund.Pedersen@regionh.dk; danlieb@fas.harvard.edu 
Classical signalling

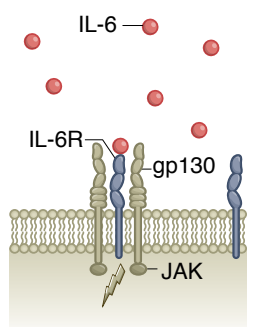

Select cell types and muscle) (especially liver

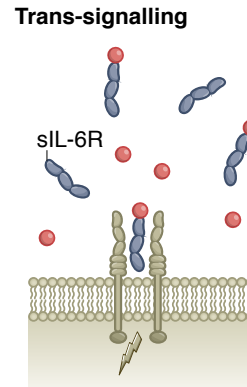

Multiple cell types
Cluster signalling

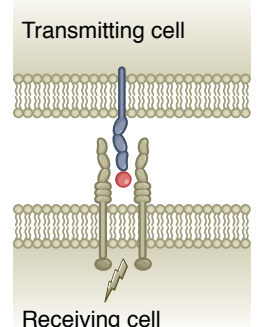

Signalling between leukocytes
Fig. 1 | The three signalling pathways of IL-6. Classical signalling relies on the interaction of the membrane-bound IL-6 receptor (IL-6R) and circulating IL-6. The resulting complex then associates with ubiquitously expressed gp130 to produce an intracellular signal through Janus kinases (JAK). IL-6R expression is restricted to a few cell types, including myocytes, hepatocytes and adipocytes, thus limiting the effects of classical signalling. Trans-signalling occurs when IL-6R is converted to a soluble receptor (sIL-6R) which can bind circulating IL-6. The resulting sIL-6R-IL-6 complex then associates with gp130 to signal intracellularly. Therefore, trans-signalling is much more widespread and can occur in any cell type that expresses gp130, such as endothelial cells, osteoclasts, myocytes or hepatocytes. Because sIL-6R production is associated with enzymatic cleavage or alternative splicing that occurs in activated innate immune cells, trans-signalling is commonly thought to potentiate the proinflammatory effects of IL-6. A further layer of regulation is conferred by soluble versions of gp130 (not pictured) that block the sIL-6R-IL-6 complex from signalling intracellularly. The last mode of IL- 6 signalling is cluster signalling. Cluster signalling occurs through presentation of the IL-6-IL-6R complex from a transmitting cell (for example, dendritic cell) to a receiving cell that expresses gp130 (for example, T cell). This signalling modality is primarily used for signalling between immune cells.

pregnancy and subsequent lactation on top of baseline maintenance and activity costs. Numerous studies of gonadal steroid hormones, proxies of long-term energy allocation to reproduction, support the hypothesis that energy allocation to reproduction is sensitive to energy availability in humans and is delayed or reduced by weight loss or high levels of $\mathrm{PA}^{29,30}$. However, in the short-term-sometimes at a moment's notice-organisms need to sense energetic stress caused by PA or an immune response and divert energy rapidly in order to survive.

Although the tradeoffs and molecular mediators of long-term energy allocation are well known, the molecules that regulate short-term energy allocation and tradeoffs that it entails are comparatively understudied. Because the timescales are so rapid, on the scale of minutes to days, we predict that short-term energy allocators share a few defining characteristics. One, they have short half-lives to limit the duration of energy allocation away from reproduction. Two, they rely on liberating stored energy and temporarily suppressing dispensable functions like digestion to meet the acute energetic demand. The most well known of these allocators are hormones and catecholamines such as cortisol and epinephrine that are secreted in response to short-term energetic stressors like prolonged PA or immune activation. Here, we propose adding IL- 6 to that list.

\section{Short-term energy allocation by IL-6}

Previous attempts to explain the confusingly pleiotropic functions of IL-6 have largely depended on mechanistic explanations. Due to the observation that enzymatic cleavage of IL-6R, the primary form of sIL-6R generation, is upregulated in activated and apoptosing neutrophils as well as in the presence of $\mathrm{CRP}^{31,32}$, it has been suggested that the dichotomy between trans- and classical signalling can partly explain the diverse context-dependent effects of IL-6, especially with respect to its pro-/anti-inflammatory dichotomy ${ }^{10}$. Still, the differences between trans- and classical signalling explain only how IL-6 can have context-dependent effects and not why a cytokine has many functions beyond the immune system.

We propose that the explanation lies in IL-6's ability to coordinate short-term energy allocation, especially during sustained, strenuous endurance PA. IL-6 is a good candidate for a short-term energy allocator for several reasons. Like other short-term energy allocators, IL-6 has a relatively short half-life of $\sim 60$ minutes during recombinant infusion in rodents ${ }^{33}$. Intriguingly, the half-life in humans may be context dependent; it is longer for IL- 6 produced by sepsis or surgical recovery ( 100 minutes- 15.5 hours $)^{34,35}$ and shorter for myokine IL-6 (5 minutes) ${ }^{36}$. Myokine IL-6 also fulfills three main roles that are characteristic of a short-term energy allocator. First, myokine IL-6 production during PA is dependent on metabolic stress within the muscle ${ }^{37}$. Second, myokine IL- 6 mobilizes stored somatic energy from adipose and skeletal muscle, primarily by promoting lipolysis ${ }^{23}$. Lastly, myokine IL-6 promotes differential uptake of liberated somatic energy in myocytes by increasing muscular insulin sensitivity ${ }^{38}$ and transiently downregulating inflammation (Fig. 2).

\section{IL-6 is a signal of energetic stress}

We have previously proposed that myokine IL- 6 acts as an energy sensor for muscle ${ }^{37}$. Early work showed that myokine IL-6 is secreted directly from myocytes and is highly sensitive to PA stimulus-increasing up to 100 -fold from baseline levels of $<5 \mathrm{pg} / \mathrm{mL}^{17}$. In addition, unlike leukocyte-derived IL-6, transcription and subsequent secretion of myokine IL- 6 occur in the absence of TNF- $\alpha$ and IL-1 $\beta$ secretion $^{39}$ (Fig. 3). During a sustained bout of moderate to vigorous PA, plasma IL-6 levels increase exponentially and reach their peak immediately after PA cessation ${ }^{40}$. The magnitude of the increase depends strongly on PA duration, intensity and the muscle mass engaged in $\mathrm{PA}^{17}$, factors that also regulate the degree of energy expenditure. After PA, increased hepatic clearance ${ }^{41}$ plus a cessation of myokine secretion cause plasma IL-6 levels to decline back to baseline in a matter of hours. Aside from myokine IL-6 kinetics during PA, the molecular regulation of secretion provides evidence that myokine IL-6 is an energy sensor.

IL-6 production during PA is primarily controlled at the level of transcription. Various stress-related molecular mechanisms are thought to control IL-6 mRNA transcription during exercise, but we will highlight only a few here. $\mathrm{Ca}^{2+}$ signalling appears to play a large role in contraction-induced IL- 6 mRNA transcription. Repeated membrane depolarizations increase intracellular and nuclear $\mathrm{Ca}^{2+}$ through activation of the PI3K cascade ${ }^{42}$. Ultimately, increased $\mathrm{Ca}^{2+}$ signalling drives IL-6 transcription by activating transcription factors including activator protein 1 (AP-1) $)^{42}$. Membrane depolarization can also increase phosphorylation on c-Jun N-terminal kinase (JNK), which also activates AP-1 (ref. ${ }^{43}$ ). However, if exercise continues, IL-6 production relies on signals of energetic stress, such as muscle glycogen depletion ${ }^{44}$, lactic-acid accumulation ${ }^{45}$ and redox signalling ${ }^{46}$. In one study, participants who pre-emptively depleted muscle glycogen in one leg before a two-legged extensor exercise exhibited greater IL-6 mRNA transcription and myokine IL-6 release in the glycogen-depleted leg $^{44}$. The mechanisms underlying this effect remain inconclusive but may involve loss of glycogen inhibition on AMPK signalling ${ }^{47,48}$ or nuclear phosphorylation of p38 MAPK at low glycogen levels ${ }^{49}$.

Experimentally manipulating energy flow towards a muscle during PA can also impact myokine IL-6 release. Increasing the energetic deficit by blocking lipolysis increases myokine IL-6 (ref. ${ }^{50}$ ) while reducing the energetic deficit through continuous carbohydrate supplementation attenuates secretion ${ }^{51}$. Furthermore, when 
Table 1 | Multifunctionality of IL-6

\begin{abstract}
Metabolic Functions
Promote catabolism

Induces lipolysis and free fatty acid release from adipocytes and skeletal muscle $^{23,55,56}$

Chronic administration contributes to insulin resistance in liver, adipose and muscle ${ }^{19,20,120}$

Induces cortisol secretion $61,122,123$

Increases blood glucose during exercise $^{58}$
\end{abstract}

Immune functions

Proinflammatory

Enhances B-cell differentiation and antibody production

Biases T-cell differentiation towards $T_{H} 2$ and $T_{H} 17$ while inhibiting $T_{H} 1$ and $T_{\text {reg }}$ formation ${ }^{8,126-128}$

Regulates acute-phase protein production (C-reactive protein, serum amyloid a, fibrinogen) and body temperature elevation ${ }^{129,130}$

Increases haematopoietic stem cell and myeloid cell production in bone marrow $^{132,133}$

Acts as a chemotactic agent for monocytes, macrophages and T cells 84,136

\section{Musculoskeletal Functions}

Catabolic

\section{Anabolic}

Mediates muscle protein breakdown when chronically elevated ${ }^{91,137}$

Induces osteoclastic differentiation and is associated with bone loss from estrogen withdrawal ${ }^{124}$

\section{Promote anabolism}

Increases insulin secretion/ upregulates GLP-1 production $22,24,65$

Acute administration increases insulin sensitivity in muscle $e^{67,21}$

Increases GH hormone secretion from the pituitary ${ }^{124}$

May increase fatty acid uptake in skeletal muscle ${ }^{64,69}$

\section{Anti-inflammatory}

Regulates neutrophil trafficking at infection site by downregulating chemokine production and increasing apoptosis ${ }^{125}$

Inhibits proinflammatory cytokines (TNF- $\alpha$ and IL-1 $\beta$ ) and increases anti-inflammatory cytokines production (IL-10 and IL-1RA) ${ }^{80,81,22}$

Sensitizes macrophages to M2 polarization and enhances $M 2$ phenotype

Regulates tissue repair and regeneration in the intestinal epithelium and liver ${ }^{134,135}$

Skeletal muscle hypertrophy via satellite cell proliferation and differentiation ${ }^{74}$

Induces early osteoblastic differentiation and may engender bone formation ${ }^{138}$

Select effects of IL- 6 on various organ systems throughout the body. IL- 6 exhibits a variety of contradictory metabolic, immune and musculoskeletal functions that largely differ depending on how long IL-6 levels are elevated. With the exception of the immunological effects, the effects described are largely confined to IL-6 administration in healthy humans and animals.

the muscle becomes more energetically efficient during training, myokine IL-6 release is sharply reduced. In one study, the IL-6 response to $\mathrm{PA}$ after a 10 -week training regime was not significantly different than the pre-training exercise stimulus despite a $44 \%$ increase in absolute workload ${ }^{52}$. Altogether, it is clear that myokine IL-6 secretion during PA is tied to intramuscular energy demands.

\section{IL-6 mobilizes stored somatic energy}

Once released, myokine IL-6 fulfills the second characteristic of a short-term energy allocator by liberating energy from somatic stores to meet muscular energetic demands. The most prominent such mechanism is lipolysis. Infusions of IL-6 in resting individuals at doses $(>100 \mathrm{pg} / \mathrm{mL})$ similar to those measured in sustained aerobic exercise $\left(\sim 80 \mathrm{pg} / \mathrm{mL}^{53,54}\right)$ increase lipolysis in both adipose and muscle tissue ${ }^{23,55,56}$. To study the effects of myokine IL-6 on energy liberation in exercising humans, researchers often use tocilizumab to inhibit IL-6 signalling. A recent study using this approach showed that tocilizumab administration during a 90-minute bout of exercise reduced fatty acid mobilization in both men who are lean and who present with obesity ${ }^{57}$. In addition, a small long-term human study that gave participants $(n=13)$ tocilizumab during a 12-week bicycle training intervention demonstrated that IL-6 inhibition prevented visceral fat loss observed in the placebo exercising group $(n=14)$ and resulted in visceral fat gain comparable to that in non-exercising control participants ${ }^{56}$.

Beyond liberating energy from fat, myokine IL-6 may also increase glucose supply in the bloodstream to fuel sustained PA. The most compelling evidence for this effect comes from a study that manipulated IL-6 levels during low- and high-intensity PA. Study participants cycled for 2 hours on 3 separate occasions at high intensity ( $70 \%$ maximal oxygen consumption $\left.\left(\mathrm{VO}_{2} \max \right)\right)$, at low intensity $\left(40 \% \mathrm{VO}_{2} \max \right)$ and at low intensity with exogenous IL-6 infusion to mimic the systemic IL-6 level during high-intensity PA. During the condition with IL-6 infusion, rates of glucose appearance and glucose disposal both increased ${ }^{58}$, suggesting that IL-6 contributes to gluconeogenesis as well as glucose disposal during exercise, a likely adaptation to enhance energy allocation towards muscle during intense PA.

In addition to the aforementioned direct pathways, myokine IL-6 liberates energy indirectly through the actions of other hormones. Several older studies found that acute injections of high doses of recombinant IL-6 (ranging from 594 to $3,500 \mathrm{pg} /$ $\mathrm{mL}$ ) in resting individuals indirectly increased blood glucose levels by inducing transient secretion of glucagon ${ }^{59,60}$. However, more recent studies with lower doses (from 42 to $220 \mathrm{pg} / \mathrm{mL}$ ) found no effect of recombinant IL-6 infusion on blood glucose appearance despite increasing glucagon levels ${ }^{55,61}$. In addition, receptors for IL- 6 are expressed in both the adrenal and pituitary glands ${ }^{62}$, indicating that myokine IL- 6 may orchestrate the production of several influential metabolic hormones, especially cortisol. Studies show that IL-6 infusions in resting individuals at physiological concentrations promote cortisol secretion ${ }^{61}$. Thus, it is possible that myokine IL- 6 contributes to the cortisol release commonly observed during PA. Lastly, it is important to note that adipocyte-derived IL-6 appears to support lipolysis as well, especially after physical activity (Box 1).

\section{IL-6 shunts energy towards exercising muscle}

The final step in myokine-IL-6-directed short-term energy allocation is the augmentation of energy uptake in skeletal muscle cells and the suppression of energy uptake by other tissues. To explore this role of myokine IL-6, we first evaluate its effect on energy uptake by muscle before turning to its inhibitory effects on other organ systems.

During and after PA, muscular glucose uptake and insulin sensitivity are enhanced to meet the energetic demands of muscle contraction and muscle recovery ${ }^{63}$ and evidence from humans and rodents suggests that myokine IL- 6 contributes to heightened insulin sensitivity after PA. Global IL-6 knockout (IL-6KO) mice have acute impairments in muscle glucose uptake after exercise and fail to show improvements in diet-induced insulin resistance after an exercise training regime ${ }^{64}$. In humans with obesity and type 2 diabetes, myokine IL-6 has been shown to enhance glucagon-like peptide 1 secretion from pancreatic alpha cells ${ }^{22,65}$, potentially contributing to the hyperinsulinemia observed in healthy controls after intense PA ${ }^{66}$. However, the duration of IL-6 signalling influences whether IL-6 has beneficial or detrimental effects. A meta-analysis of 10 studies found that chronically high levels of IL-6 were associated with the development of type 2 diabetes $^{18}$. Murine and cell-based studies have found that IL- 6 signalling for more than 


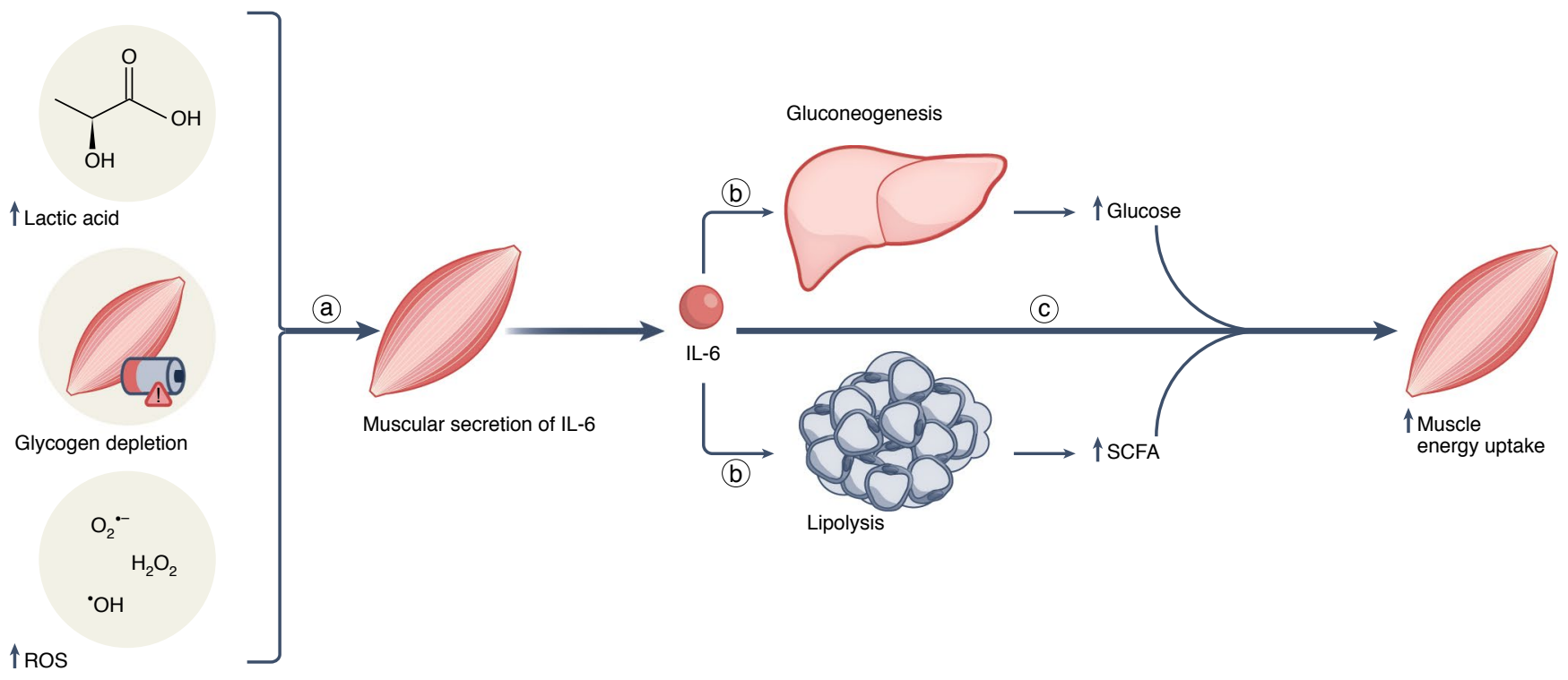

Fig. 2 | Myokine IL-6 as a short-term energy allocator during physical activity. a, Energy sensing. Physiological components of prolonged muscle activity, such as lactic-acid buildup and reactive oxygen species (ROS) production along with muscle glycogen depletion, signal that intramuscular energy stores are depleted and precipitate myokine secretion of IL-6. b. Energy liberation. When myokine IL-6 is released into circulation, it liberates somatic energy for the contracting muscle by upregulating lipolysis and gluconeogenesis throughout the body. In addition, myokine IL- 6 acts indirectly to increase catabolism and energy mobilization by inducing cortisol secretion (not pictured). c, Energy allocation. At the same time, myokine IL-6 increases energy uptake by the muscle through increases in insulin receptor sensitivity, GLUT4 expression and possibly fatty acid transporter expression. Evidence suggests that it also allocates energy to the muscle indirectly by transiently downregulating other energy-consuming processes, such as the immune activity (not pictured). The energy allocated towards the muscle by IL-6 during and after activity is used to fuel muscle contraction and is likely important for muscle recovery and repair. SCFA, short-chain fatty acids.

24 hours induces insulin resistance in adipose and hepatic tissue $\mathrm{e}^{19,20}$. Conversely, other experiments show that doses of IL- 6 similar in magnitude to those measured during PA promote muscular glucose uptake through insulin receptor substrate 1 or AMP-activated protein kinase (AMPK) activation ${ }^{21,67,68}$.

AMPK activation is crucial for another mechanism of IL-6-mediated glucose uptake: GLUT4 translocation to the sarcolemma. In vitro, IL-6 stimulates GLUT4 translocation in a dose-dependent manner through activation of AMP-activated protein kinase $(\mathrm{AMPK})^{67}$. However, in rodents, myokine IL-6 appears to have negligible effects on contraction-mediated insulin-independent GLUT4 translocation as GLUT4 levels are similar between IL-6KO and wild-type animals immediately after $\mathrm{PA}^{69}$. Despite this, exercised mice injected with IL-6 neutralizing antibody display impaired GLUT4 expression and glucose uptake 24 hours after activity ${ }^{38}$. This impairment suggests that myokine IL-6 is particularly important for muscle recovery by regulating muscular glucose uptake after activity.

Lipids are another important energy source during PA, and although most lipid transport is conventionally assumed to occur by diffusion, fatty acid uptake can be acutely regulated by the relative expression of fatty acid transporters ${ }^{70}$. As was the case with glucose transporters, IL-6 may mediate some of the increases in fatty acid transporter expression and translocation seen during $\mathrm{PA}^{71}$. Studies have found that fatty acid transporter expression is impaired after PA in muscle-specific (IL-6MKO) and IL-6KO mice, reducing fatty acid transport into the muscle ${ }^{64,69}$. Additionally, myokine IL-6 may limit fatty acid uptake by adipocytes if it acts similarly to recombinant IL-6, which downregulates lipoprotein lipase in mouse adipocytes $^{72}$. These results raise the possibility that myokine IL-6 may upregulate muscle energy uptake not only through glucose uptake but also through fatty acid absorption.
One way to test whether myokine IL- 6 allocates energy towards the muscle is to investigate its effects on PA performance and recovery. If myokine IL-6 increases energy uptake through the muscle, any disruption should reduce performance and impair muscle recovery and adaptation. In IL-6MKO and IL-6KO mice, these deficits in performance are readily observable. Despite similar maximal exercise intensity and daily overall activity levels between control and IL-6KO strains ${ }^{73}$, knockout mice fatigue faster when running at a submaximal intensity until exhaustion ${ }^{64,69}$.

Few studies have examined how myokine IL-6 deficiency impacts muscular recovery and adaptation to repeated exercise bouts, but there are clues that the downstream effects of myokine IL-6 are as important after PA as they are during PA. The first major benefit of myokine IL-6 is the transient anti-inflammatory environment induced after $\mathrm{PA}^{39}$. In addition, IL-6 directly regulates muscle hypertrophy ${ }^{74}$, possibly through the induction of IL-10 and enhanced polarization of M2 macrophages ${ }^{75}$. In fact, genetic polymorphisms in the promoter region of the IL6 gene that increase IL-6 levels have been linked to increased fat-free mass in men ${ }^{76}$. Lastly, IL-6-STAT3 signalling may contribute to some muscular adaptations that occur after training, such as the induction of mitochondrial biogenesis and increased mitochondrial activity ${ }^{77}$ driven by PGC-1 $\alpha$ (ref. $\left.{ }^{78}\right)$. Intriguingly, this effect appears to be restricted to specific muscle tissues ${ }^{79}$.

In addition to regulating energy uptake and possible repair within the muscle, IL-6 has inhibitory effects on the immune system, which allows the muscle to use more of the liberated somatic energy during and after PA. In particular, myokine IL-6 inhibits monocyte production of TNF- $\alpha$ (ref. ${ }^{80}$ ) and possibly IL- $1 \beta$ (ref. ${ }^{81}$ ) while inducing the expression of major anti-inflammatory cytokines ${ }^{54}$, namely interleukin 10 (IL-10), interleukin 1 receptor antagonist (IL-1ra) and soluble TNF receptor. IL-10 is especially important 

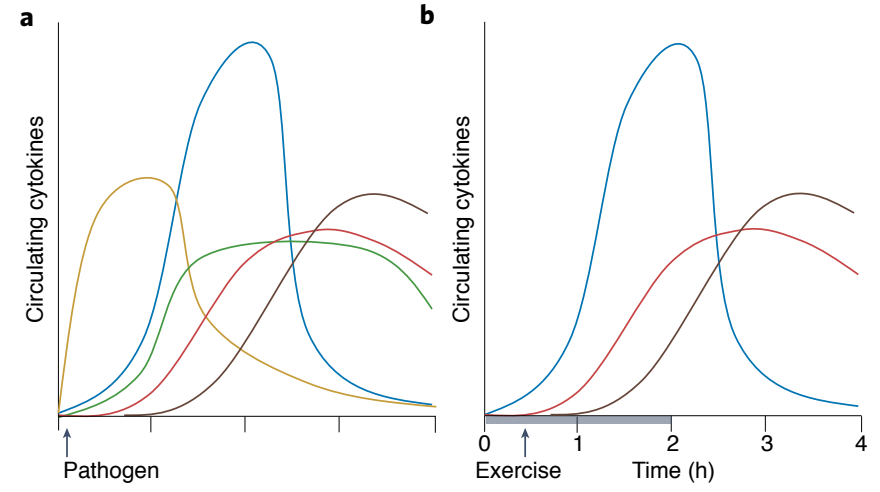

$\begin{array}{llll}\text {-TNF- } \alpha & -\mathrm{IL}-6 \quad-\mathrm{IL}-10 & -\mathrm{TNF} \text { receptor }\end{array}$

Fig. 3 | Cytokine secretion during an immune response versus myokine secretion during exercise. $\mathbf{a}$, In the acute-phase immune response, pathogenic proteins activate the innate immune system and initiate a NF- $\kappa B$-mediated cytokine response involving TNF- $\alpha$, IL-1 and IL-6. b, In contrast, myokine IL- 6 secretion is induced in the absence of TNF- $\alpha$ and IL-1, leading to a net anti-inflammatory benefit. This figure was adapted, with permission, from ref. ${ }^{139}$.

in energy allocation because, in addition to limiting immune cell activity, muscle-specific IL-10 directly increases glucose uptake ${ }^{82}$.

\section{IL-6 in chronic inflammation and metabolic syndrome}

IL-6 is most commonly considered a proinflammatory cytokine involved in the acute-phase immune response and chronic inflammation, but an evolutionary perspective on IL-6's function in short-term energy allocation sheds new light on its roles in modern post-industrial environments. Many people today in high-income countries engage in very low levels of moderate to vigorous PA (MVPA)-less than $5 \%$ of US adults meet the World Health Organization's recommendation of 30 minutes per day at least 5 days per week ${ }^{83}$-and have almost unlimited access to energy-rich food. Because hunter gatherers and nonindustrial agricultural populations have high levels of MVPA and fluctuating energy availability, we can infer that it used to be normal for individuals to regularly stimulate myokine IL-6 secretion in order to direct energy towards muscle. In the absence of PA-induced energetic stress to stimulate myokine IL-6 secretion, more energy is stored as fat that in turn drives chronic inflammation. Loss of regular myokine IL-6 secretion in modern environments thus contributes to a mismatch by not only increasing energy storage in adipocytes, but also failing to stimulate the production of anti-inflammatory myokines.

Our proposed model of IL- 6 as an energy allocation can also be used to reinterpret IL- 6 secretion during another modern phenomenon: chronic IL-6 secretion from leukocytes or stromal cells. During the course of an infection, IL- 6 mediates the transition from granulocyte invasion to monocyte and lymphocyte recruitment in inflamed tissue ${ }^{84}$, representing a switch from damaging inflammation to long-lasting, targeted pathogen defence. However, when IL-6 is secreted chronically by tissues, it contributes to chronic inflammation and immune dysregulation. For example, in the presence of another proinflammatory cytokine, IL-17, excessive IL-6 stimulation of stromal cells induces a positive feedback loop of NF-kB and STAT3 activation, which leads to increased chemokine production and prolonged local inflammation ${ }^{85}$. This loop, termed the IL- 6 amplifier, is thought to be involved in the pathogenesis of several autoimmune diseases ${ }^{86}$. Chronic IL- 6 also supports autoimmunity and inflammation by biasing the differentiation of naive $\mathrm{T}$ cells towards type $17 \mathrm{~T}$ helper $\left(\mathrm{T}_{\mathrm{H}} 17\right)$ cells at the expense of anti-inflammatory regulatory $\mathrm{T}\left(\mathrm{T}_{\mathrm{reg}}\right)$ cells $\mathrm{s}^{87}$. In the bone marrow,

\section{Box 1 | Adipose tissue IL-6}

White adipose tissue (WAT) is one of the major sources of IL-6 production at rest. During obesity, IL-6 production predominantly occurs from visceral WAT, whereas subcutaneous WAT is the primary source in lean individuals ${ }^{140}$. Similar to myokine IL-6, adipocyte-derived IL-6 is sensitive to energetic stress. In visceral WAT of obese rodents, necrotic and hypertrophic adipocytes induce IL-6 secretion by surrounding myeloid cells and adipocytes ${ }^{101}$. In subcutaneous WAT, intracellular signals of energetic stress, such as cAMP levels, appear to induce IL6. Increasing CAMP levels through inhibition of obesity-driven non-canonical IKK- $\varepsilon$ and TBK1 signalling significantly increases plasma IL-6 values, which then go on to inhibit hepatic gluceoneogenesis ${ }^{141}$. Further evidence comes from an in vitro study that exposed human adipocytes to $\beta$ adrenergic receptor stimulation, a potent mechanism to increase CAMP $^{142}$, and observed a sixfold increase in IL-6 secretion ${ }^{143}$.

In the case of visceral WAT IL-6, our model predicts that IL-6 increases lipolysis and possibly insulin resistance (see discussion in the main text) to generate fuel for an inflammatory response. In contrast, subcutaneous WAT IL- 6 may be induced to further stimulate lipolysis in response to fasting or physical activity. This is supported by increases in subcutaneous WAT IL-6 during exercise recovery ${ }^{144}$. We note, however, that insulin can also stimulate IL-6 production from adipocytes in vitro through $\mathrm{cGMP}^{145}$, suggesting that more research is needed to understand the depot-specific functions of adipocyte IL-6.

IL-6 enhances myelopoiesis ${ }^{88}$ and increases neutrophil mobilization into circulation ${ }^{89}$, suggesting that chronically high levels of IL-6 sustain leukocyte recruitment into inflamed tissues.

From an energetic perspective, immune activation imposes a large metabolic burden on the body both acutely and chronically. It has long been recognized that inflammation regulates short-term energy allocation towards the immune system through insulin resistance ${ }^{90}$. However, the identity of the cytokines that direct short-term energy allocation during inflammation is still an open question. On the basis of our model of IL-6, we hypothesize that IL-6 helps to maintain short-term energy allocation towards the immune system during chronic inflammation. To evaluate this hypothesis, we now turn to the metabolic role of IL-6 in chronic inflammatory diseases, such as RA or Castleman disease. For example, basal elevations of IL-6 induce weight loss and cachexia in RA. IL-6, in combination with other proinflammatory cytokines such as TNF- $\alpha$ and IL- $1 \beta$, promotes muscle catabolism ${ }^{91}$, which may support the ongoing immune response. Further support for the hypothesis of IL-6 as an energy allocator during chronic inflammation comes from data on the metabolic changes that occur during IL- 6 blockades that are used to treat people with RA and Castleman disease.

\section{Metabolic changes during therapeutic IL- 6 blockades}

The use of IL-6 receptor blockade is an indisputable breakthrough treatment therapy for RA and other inflammatory diseases. However, it is also well documented that administration of IL-6 pathway inhibitors is associated with an increase in body weight ${ }^{92}$ and lean mass ${ }^{91,93}$ in individuals with RA and Castleman disease. Importantly, one study in people with RA documented a shift in body fat distribution, characterized by a decrease in visceral fat and increased subcutaneous fat ${ }^{11}$. Given that subcutaneous fat is less active, this raises the possibility that IL- 6 is shuttling body fat into readily accessible depots. Furthermore, IL- 6 blockades often result in an increase in total and low-density lipoprotein cholesterol ${ }^{93,94}$. Importantly, this effect is due to a decrease in serum cholesterol 


\section{Box 2 | Energy allocation by IL-6 in the context of cancer}

Cancer is another context in which IL-6 may act to allocate energy. Because cancer cells compete with other cells for energy, we hypothesize that cancer cells sometimes co-opt short-term energy-allocation mechanisms, such as IL-6, to gain continued access to somatic energy. In support of this view, elevated IL-6 levels are present in the majority of people with cancer, fuelling uncontrolled cell growth and angiogenesis ${ }^{146}$. Cancer-derived IL-6 responds to cellular energy balance, as treatment with metabolic inhibitors upregulates transcription and secretion of IL-6 in multiple cancer lines ${ }^{147}$. In another parallel to its role during PA, cancer-derived IL-6 liberates energy and contributes to cancer-related cachexia ${ }^{137}$. Finally, IL- 6 produced by tumours directs energy towards cancer cells in certain contexts. During breast cancer, a study found that IL- 6 aids cancer cells in evading anti-vascular endothelial growth factor treatment by promoting resistance to hypoxia ${ }^{148}$. Although more work is needed, this suggests that IL-6 may be important in sustaining cancer proliferation.

catabolism $^{95}$, rather than an increase in cholesterol production. Lastly, a recent study reported that tocilizumab administration may ameliorate insulin resistance and decrease $\mathrm{HbAlc}^{96}$, but these results could not be replicated ${ }^{91,93}$. These effects of IL-6 receptor blockade in humans are generally compatible with observations in experimental models, demonstrating that IL-6-deficient mice develop mature-onset obesity ${ }^{97}$. A perfect match between the effects of an IL- 6 blockade in humans and the phenotype of IL-6KO mice is unlikely because human studies have a different baseline metabolism due to underlying disease inflammation. Despite this, data from IL-6 blockades support the hypothesis that IL-6 is crucial in providing the energy, particularly cholesterol and fat, to fuel chronic inflammation in RA. We do note however, that while IL-6 blockades may inhibit IL- 6 based energy allocation towards the immune system, they also might detrimentally affect the metabolic benefits of exercise training in these patients ${ }^{56}$.

\section{IL-6 as an energy allocator in metabolic syndrome}

Aside from inflammatory diseases, elevated systemic IL-6 often co-occurs with obesity and metabolic syndrome but the role of IL-6 in metabolic disease remains controversial. To determine whether obesity-induced IL- 6 can be characterized as an energy allocator, we need to ask whether chronically elevated IL- 6 is a marker or a cause of obesity-related insulin resistance. In both rodents and humans with obesity, necrotic and hypoxic adipocytes induce tissue-resident leukocytes and other adipocytes to secrete a milieu of proinflammatory cytokines, including IL-6, TNF- $\alpha$ and IFN- $\gamma$, that initiates further leukocyte infiltration of adipose tissue and the development of chronic inflammation ${ }^{98}$. This has led to the hypothesis that increased levels of circulating or tissue IL-6 in obesity is a cause of insulin resistance ${ }^{19,99}$. Studies on the direct effects of IL-6 in adipose tissue from obese mice indicate that different sources of IL-6 drive opposing phenotypes with respect to insulin resistance (Box 1). For example, IL-6 signalling in adipose tissue T cells and adipocyte IL-6 secretion appear to exacerbate insulin resistance ${ }^{100,101}$, whereas macrophage IL-6 is insulin-sensitizing ${ }^{101,102}$. The data on the effect of adipocyte-specific IL-6 in genetically obese $(o b / o b)$ mice are not consistent, as studies both support ${ }^{101}$ and undermine $e^{103}$ the assertion that adipocyte IL-6 leads to insulin resistance. In humans, a recent report suggests that administration of tocilizumab in people with obesity reduces HOMA-IR ${ }^{104}$. In total, there is no definitive consensus on whether IL-6 from adipose tissue derived from individuals with obesity leads to insulin resistance and allocates energy to

\section{Box 3 | Key predictions of the IL- 6 energy allocation model}

- IL-6 release by a tissue is regulated by stress, particularly metabolic stress. In exercising muscle, the magnitude of IL-6 release is primarily determined by signals of energetic depletion within the tissue.

- IL-6 liberates stored somatic energy for immediate use. During exercise, IL- 6 liberates fat and glucose for use by the muscle in order to prevent extreme depletion of intramuscular energy reserves.

- IL-6 upregulates energy transport proteins of the tissue that secreted it, and inhibits energy uptake in other peripheral tissues. Myokine IL-6 increases the expression of glucose and fatty acid transporters on the muscle membrane and enhances muscle insulin sensitivity while impairing energy uptake in other tissues.

- Disruptions in myokine IL-6 signalling will impair the ability of the muscle to restore its energy reserves during recovery from exercise. By controlling the flow of energy towards the muscle during recovery, IL-6 will affect muscular adaptation to and metabolic benefits of exercise.

- Chronic IL-6 blockades during chronic inflammation or cancer will limit energy uptake by leukocytes and cancer cells, respectively, and will reverse cachexia.

adipose tissue inflammation. One reason for which obesity-induced IL-6 may not fit our model is that obesity is characterized by vast energy storage and is frequently associated with positive energy balance. As IL-6-directed short-term energy allocation is predicated on an energy deficit, IL-6 secreted within the context of obesity may play a different role.

\section{IL-6 and COVID-19}

In addition to its role in chronic inflammation, IL-6 plays a key role in initiating and sustaining acute inflammation, as recently highlighted by COVID-19. In response to COVID-19 infection, IL- 6 acts as a proinflammatory cytokine, and baseline IL-6 levels are prognostic of disease severity ${ }^{105}$. Mechanistically, IL-6 plays a key role in COVID-19-associated hyperinflammation, which exacerbates acute respiratory distress syndrome and multiorgan failure ${ }^{106}$. High levels of IL-6 are especially seen in COVID-19-infected individuals with chronic infection that is accompanied by chronic low-grade inflammation ${ }^{107}$. This subgroup appears to have an impaired ability to mount a sufficient anti-inflammatory response, leading to a cytokine storm. Accordingly, treatment with IL-6 receptor antagonists, most commonly tocilizumab, has been demonstrated to lower all-cause mortality for hospitalized people with COVID-19 (ref. ${ }^{108}$ ). However, IL- 6 blockades may be effective only in those with severe disease $^{109}$, suggesting that IL-6-based energy allocation is relevant only to limit hyperinflammation.

\section{Conclusions and questions for future research}

The energy-allocation model of IL-6 helps to make sense of this molecule's disparate and seemingly contradictory roles. Secreted in response to energetic stress within a tissue, IL-6 liberates stored somatic energy and directs that energy towards the tissue in question while downregulating energy expenditure elsewhere (Fig. 2). This framework helps to explain why IL- 6 can have both beneficial and detrimental effects on human health. In the short-term, it can allocate energy towards life-saving immune responses or a burst of PA. Chronically, that same energy-allocation mechanism can continually shunt resources to an activated immune system or cancer (Box 2), attenuating beneficial anabolic processes. 
Despite its important role in short-term energy allocation, we acknowledge that IL- 6 is not responsible for all aspects of short-term energy allocation during PA or other contexts involving acute energetic stress. The high costs of failing to meet energy demands during PA likely drove the evolution of multiple redundant mechanisms, including PA-induced glucocorticoids and catecholamines, that rapidly mobilize and divert energy towards contracting muscle while restricting reproductive and immune function ${ }^{110}$. Other myokines, such as IL-15 and IL-13, are thought to act locally to enhance muscle insulin sensitivity and metabolic adaption to exercise ${ }^{111,112}$. Therefore, further research is needed to explore potential intracellular crosstalk between these myokines and to determine whether they can potentially compensate for one another to meet energetic demands within muscle.

Despite many lines of support in human and mouse data, there are multiple conflicting reports regarding the role of IL-6-induced energy liberation during exercise. The bulk of these data come from rodent models of myokine IL- 6 secretion, which are discordant with human trials. For example, several rodent studies report that IL-6KO and IL-6MKO mice have similar glucose mobilization and muscle glucose uptake during activity when compared with wild-type mice ${ }^{113-115}$. Only two studies have measured changes in plasma fatty acids in these animals, and they similarly found little difference in fat mobilization ${ }^{114,116}$. That said, many studies have also found that a lack of myokine IL- 6 leads to increased carbohydrate utilization and glycogen depletion ${ }^{69,113,117}$, suggesting that these animals are compensating for deficient access to stored somatic energy. The apparent defect in IL-6-induced energy mobilization is puzzling as we might expect myokine IL-6 to coordinate short-term energy allocation in the broad range of animals that produce IL-6. One potential explanation for this is the difference between mice and humans with regards to the contribution of liver and muscle to whole body metabolism. IL-6 has been shown to activate suppressor of cytokine signalling (SOCS) proteins in the liver, leading to hepatic insulin resistance ${ }^{118}$. Although IL-6 weakly increases SOCS3 expression in muscle ${ }^{68}$, it potently activates AMPK signalling ${ }^{67}$. As muscle tissue dominates in humans, the possibility exists that the negative effects of IL-6 on SOCS3 in the liver may be overridden by the positive effects on muscular AMPK.

From an evolutionary perspective, a difference in the myokine IL- 6 function between humans and mice is expected given that there was selection for increased levels of sustained endurance PA in the human lineage ${ }^{119}$. IL-6-directed mobilization of stored somatic energy reserves makes sense only if an animal is active enough to exhaust muscular energy reserves. Since wild mice are unlikely to exhaust their glycogen stores during PA in the wild, IL-6 may only serve as a marker of energetic stress. Given the possibility of many redundant mechanisms for energy liberation during $\mathrm{PA}$, it is also possible that IL-6 is sufficient, but not required, for energy mobilization in these animals. Altogether, more mechanistic studies of myokine IL- 6 function are needed to determine whether and how IL-6-directed energy mobilization and allocation differ between humans and model organisms.

Our model also generates several predictions with respect to IL-6's functions and regulation (Box 3). Despite the focus on myokine IL-6, our model of IL-6 as an energy allocator can be applied more broadly to other contexts, such as chronic inflammatory diseases in which IL-6 is secreted by other tissues. In these cases, high levels of IL-6 seem to liberate energy through cachexia and lipolysis and may allocate energy by modulating insulin resistance. However, more data are needed to definitively conclude that IL-6 is directly upregulating metabolic pathways to increase energy uptake in immune cells. In sum, modelling IL-6 as an energy allocator elucidates how it can perform seemingly contradictory metabolic effects across the body.
Received: 25 August 2021; Accepted: 21 January 2022;

Published online: 24 February 2022

\section{References}

1. Hirano, T. et al. Purification to homogeneity and characterization of human B-cell differentiation factor (BCDF or BSFp-2). Proc. Natl Acad. Sci. USA 82, 5490-5494 (1985)

2. Tanaka, T., Narazaki, M. \& Kishimoto, T. IL-6 in inflammation, immunity, and disease. Cold Spring Harb. Perspect. Biol. 6, 16295-16296 (2014).

3. Pedersen, B. K., Åkerström, T. C. A., Nielsen, A. R. \& Fischer, C. P. Role of myokines in exercise and metabolism. J. Appl. Physiol. 103, 1093-1098 (2007).

4. Vicennati, V., Vottero, A., Friedman, C. \& Papanicolaou, D. A. Hormonal regulation of interleukin-6 production in human adipocytes. Int. J. Obes. 26, 905-911 (2002).

5. Costa, M. M., Maehr, T., Diaz-Rosales, P., Secombes, C. J. \& Wang, T. Bioactivity studies of rainbow trout (Oncorhynchus mykiss) interleukin-6: effects on macrophage growth and antimicrobial peptide gene expression. Mol. Immunol. 48, 1903-1916 (2011).

6. Gorshkova, E. A., Nedospasov, S. A. \& Shilov, E. S. Evolutionary plasticity of IL-6 cytokine family. Mol. Biol. 50, 918-926 (2016).

7. Geisterfer, M. et al. Regulation of IL-6 and the hepatic IL-6 receptor in acute inflammation in vivo. Cytokine 5, 1-7 (1993).

8. Päth, G. et al. Human breast adipocytes express interleukin-6 (IL-6) and its receptor system: increased IL- 6 production by $\beta$-adrenergic activation and effects of IL-6 on adipocyte function. J. Clin. Endocrinol. Metab. 86, 2281-2288 (2001).

9. Keller, C. et al. Effect of exercise, training, and glycogen availability on IL-6 receptor expression in human skeletal muscle. J. Appl. Physiol. 99, 2075-2079 (2005).

10. Scheller, J., Chalaris, A., Schmidt-Arras, D. \& Rose-John, S. The pro- and anti-inflammatory properties of the cytokine interleukin-6. Biochim. Biophys. Acta 1813, 878-888 (2011).

11. Lust, J. et al. Isolation of an mRNA encoding a soluble form of the human interleukin-6 receptor. Cytokine 4, 96-100 (1992).

12. Wolf, J. et al. Different soluble forms of the interleukin- 6 family signal transducer gp130 fine-tune the blockade of interleukin-6 trans-signaling. J. Biol. Chem. 291, 16186-16196 (2016).

13. Heink, S. et al. Trans-presentation of IL-6 by dendritic cells is required for the priming of pathogenic $\mathrm{T}_{\mathrm{H}} 17$ cells. Nat. Immunol. 18, 74-85 (2017).

14. Ruderman, N. B. et al. Interleukin- 6 regulation of AMP-activated protein kinase: potential role in the systemic response to exercise and prevention of the metabolic syndrome. Diabetes 55, S48-54 (2006).

15. Maggio, M., Guralnik, J. M., Longo, D. L. \& Ferrucci, L. Interleukin-6 in aging and chronic disease: a magnificent pathway. J. Gerontol. Ser. A Biol. Sci. Med. Sci. 61, 575-584 (2006).

16. Srirangan, S. \& Choy, E. H. The role of Interleukin 6 in the pathophysiology of rheumatoid arthritis. Therapeutic Adv. Musculoskelet. Dis. 2, 247-256 (2010).

17. Fischer, C. P. Interleukin- 6 in acute exercise and training: what is the biological relevance? Exerc. Immunol. Rev. 12, 6-33 (2006).

18. Wang, $X$. et al. Inflammatory markers and risk of type 2 diabetes: a systematic review and meta-analysis. Diabetes Care 36, 166-175 (2013).

19. Klover, P. J., Zimmers, T. A., Koniaris, L. G. \& Mooney, R. A. Chronic exposure to interleukin-6 causes hepatic insulin resistance in Mice. Diabetes 52, 2784-2789 (2003).

20. Rotter, V., Nagaev, I. \& Smith, U. Interleukin-6 (IL-6) induces insulin resistance in $3 \mathrm{~T} 3-\mathrm{L} 1$ adipocytes and is, like IL- 8 and tumor necrosis factor- $\alpha$, overexpressed in human fat cells from insulin-resistant subjects. $J$. Biol. Chem. 278, 45777-45784 (2003).

21. Saini, A. et al. Interleukin-6 in combination with the interleukin-6 receptor stimulates glucose uptake in resting human skeletal muscle independently of insulin action. Diabetes Obes. Metab. 16, 931-936 (2014).

22. Ellingsgaard, H. et al. Interleukin-6 enhances insulin secretion by increasing glucagon-like peptide-1 secretion from L cells and alpha cells. Nat. Med. 17, 1481-1489 (2011).

23. van Hall, G. et al. Interleukin-6 stimulates lipolysis and fat oxidation in humans. J. Clin. Endocrinol. Metab. 88, 3005-3010 (2003).

24. Timper, K. et al. IL-6 improves energy and glucose homeostasis in obesity via enhanced central IL-6 trans-signaling. Cell Rep. 19, 267-280 (2017).

25. Lang Lehrskov, L. et al. Interleukin-6 delays gastric emptying in humans with direct effects on glycemic control. Cell Metab. 27, 1201-1211.e3 (2018).

26. Muñoz-Cánoves, P., Scheele, C., Pedersen, B. K. \& Serrano, A. L. Interleukin- 6 myokine signaling in skeletal muscle: a double-edged sword? FEBS J. 280, 4131-4148 (2013).

27. Blanchard, F., Duplomb, L., Baud'huin, M. \& Brounais, B. The dual role of IL-6-type cytokines on bone remodeling and bone tumors. Cytokine Growth Factor Rev. 20, 19-28 (2009). 
28. Stearns, S. C. The Evolution of Life Histories (Oxford University Press, 1992).

29. Jasienska, G., Ziomkiewicz, A., Thune, I., Lipson, S. F. \& Ellison, P. T. Habitual physical activity and estradiol levels in women of reproductive age. Eur. J. Cancer Prev. 15, 439-445 (2006).

30. Ziomkiewicz, A., Ellison, P. T., Lipson, S. F., Thune, I. \& Jasienska, G. Body fat, energy balance and estradiol levels: a study based on hormonal profiles from complete menstrual cycles. Hum. Reprod. 23, 2555-2563 (2008).

31. Chalaris, A. et al. Apoptosis is a natural stimulus of IL6R shedding and contributes to the proinflammatory trans-signaling function of neutrophils. Blood 110, 1748-1755 (2007)

32. Jones, S. A. et al. C-reactive protein: a physiological activator of interleukin 6 receptor shedding. J. Exp. Med. 189, 599-604 (1999).

33. Castell, J. V. et al. Plasma clearance, organ distribution and target cells of interleukin-6/hepatocyte-stimulating factor in the rat. Eur. J. Biochem. 177, 357-361 (1988).

34. Waage, A., Brandtzaeg, P., Halstensen, A., Kierulf, P. \& Espevik, T. The complex pattern of cytokines in serum from patients with meningococcal septic shock. Association between interleukin 6, interleukin 1, and fatal outcome. J. Exp. Med. 169, 333-338 (1989).

35. Wirtz, D. C., Heller, K. D., Miltner, O., Zilkens, K. W. \& Wolff, J. M. Interleukin-6: a potential inflammatory marker after total joint replacement. Int. Orthop. 24, 194-196 (2000).

36. Toft, A. D., Falahati, A. \& Steensberg, A. Source and kinetics of interleukin-6 in humans during exercise demonstrated by a minimally invasive model. Eur. J. Appl. Physiol. 111, 1351-1359 (2011).

37. Pedersen, B. K. Muscular interleukin-6 and its role as an energy sensor Med. Sci. Sports Exerc. 44, 392-396 (2012).

38. Ikeda, S. et al. Exercise-induced increase in IL-6 level enhances GLUT4 expression and insulin sensitivity in mouse skeletal muscle. Biochem. Biophys. Res. Commun. 473, 947-952 (2016).

39. Petersen, A. M. W. \& Pedersen, B. K. The anti-inflammatory effect of exercise. J. Appl. Physiol. 98, 1154-1162 (2005).

40. Pedersen, B. K. Muscle as a secretory organ. Compr. Physiol. 3 , 1337-1362 (2013).

41. Febbraio, M. A. et al. Hepatosplanchnic clearance of interleukin-6 in humans during exercise. Am. J. Physiol. Endocrinol. Metab. 285, E397-E402 (2003).

42. Bustamante, M., Fernández-Verdejo, R., Jaimovich, E. \& Buvinic, S. Electrical stimulation induces IL-6 in skeletal muscle through extracellula ATP by activating $\mathrm{Ca}^{2+}$ signals and an IL-6 autocrine loop. Am. J. Physiol. Endocrinol. Metab. 306, E869 (2014).

43. Whitham, M. et al. Contraction-induced interleukin- 6 gene transcription in skeletal muscle is regulated by c-Jun terminal kinase/activator protein-1. J. Biol. Chem. 287, 10771-10779 (2012).

44. Steensberg, A. et al. Interleukin-6 production in contracting human skeletal muscle is influenced by pre-exercise muscle glycogen content. J. Physiol. 537, 633-639 (2001)

45. Hojman, P. et al. IL-6 release from muscles during exercise is stimulated by lactate-dependent protease activity. Am. J. Physiol. Endocrinol. Metab. 316 E940-E947 (2019).

46. Fischer, C. P. et al. Supplementation with vitamins $\mathrm{C}$ and $\mathrm{E}$ inhibits the release of interleukin-6 from contracting human skeletal muscle. J. Physiol. 558, 633-645 (2004).

47. Nylén, C. et al. IL6 and LIF mRNA expression in skeletal muscle is regulated by AMPK and the transcription factors NFYC, ZBTB14, and SP1. Am. J. Physiol. Endocrinol. Metab. 315, E995-E1004 (2018).

48. Polekhina, G. et al. AMPK $\beta$ subunit targets metabolic stress sensing to glycogen. Curr. Biol. 13, 867-871 (2003).

49. Chan, M. H. S., McGee, S. L., Watt, M. J., Hargreaves, M. \& Febbraio, M. A Altering dietary nutrient intake that reduces glycogen content leads to phosphorylation of nuclear p38 MAP kinase in human skeletal muscle: association with IL-6 gene transcription during contraction. FASEB J. 18, 1785-1787 (2004).

50. Holmes, A. G., Watt, M. J. \& Febbraio, M. A. Suppressing lipolysis increases interleukin- 6 at rest and during prolonged moderate-intensity exercise in humans. J. Appl. Physiol. 97, 689-696 (2004)

51. Nieman, D. C. et al. Carbohydrate ingestion influences skeletal muscle cytokine mRNA and plasma cytokine levels after a 3-h run. J. Appl. Physiol. 94, 1917-1925 (2003).

52. Fischer, C. P. et al. Endurance training reduces the contraction-induced interleukin-6 mRNA expression in human skeletal muscle. Am. J. Physiol. Endocrinol. Metab. 287, E1189-94 (2004).

53. Nehlsen-Cannarella, S. L. et al. Carbohydrate and the cytokine response to 2.5 h of running. J. Appl. Physiol. 82, 1662-1667 (1997).

54. Ostrowski, K., Rohde, T., Asp, S., Schjerling, P. \& Pedersen, B. K. Pro- and anti-inflammatory cytokine balance in strenuous exercise in humans. $J$. Physiol. 515, 287-291 (1999).

55. Wolsk, E., Mygind, H., Grøndahl, T. S., Pedersen, B. K. \& van Hall, G. IL-6 selectively stimulates fat metabolism in human skeletal muscle. Am. J. Physiol. Endocrinol. Metab. 299, 832-840 (2010).
56. Wedell-Neergaard, A. S. et al. Exercise-induced changes in visceral adipose tissue mass are regulated by IL- 6 signaling: a randomized controlled trial. Cell Metab. 29, 844-855 (2019).

57. Trinh, B. et al. Blocking endogenous IL-6 impairs mobilization of free fatty acids during rest and exercise in lean and obese men. Cell Rep. Med. 2, 100396 (2021)

58. Febbraio, M. A., Hiscock, N., Sacchetti, M., Fischer, C. P. \& Pedersen, B. K. Interleukin-6 is a novel factor mediating glucose homeostasis during skeletal muscle contraction. Diabetes 53, 1643-1648 (2004).

59. Tsigos, C. et al. Dose-dependent effects of recombinant human interleukin-6 on glucose regulation. J. Clin. Endocrinol. Metab. 82, 4167-4170 (1997).

60. Stouthard, J. M. L. et al. Endocrinologic and metabolic effects of interleukin-6 in humans. Am. J. Physiol. Endocrinol. Metab. 268, E813-E819 (1995).

61. Petersen, E. W. et al. Acute IL-6 treatment increases fatty acid turnover in elderly humans in vivo and in tissue culture in vitro. Am. J. Physiol. Endocrinol. Metab. 288, 155-162 (2005).

62. Bethin, K. E., Vogt, S. K. \& Muglia, L. J. Interleukin-6 is an essential, corticotropin-releasing hormone-independent stimulator of the adrenal axis during immune system activation. Proc. Natl Acad. Sci. USA 97, 9317-9322 (2000)

63. Richter, E. A. \& Hargreaves, M. Exercise, GLUT4, and skeletal muscle glucose uptake. Physiological Rev. 93, 993-1017 (2013).

64. Chowdhury, S. et al. Muscle-derived interleukin 6 increases exercise capacity by signaling in osteoblasts. J. Clin. Investig. 130, 2888-2902 (2020).

65. Ellingsgaard, H. et al. GLP-1 secretion is regulated by IL- 6 signalling: a randomised, placebo-controlled study. Diabetologia 63, 362-373 (2020).

66. Marliss, E. B. \& Vranic, M. Intense exercise has unique effects on both insulin release and its roles in glucoregulation: implications for diabetes. in Diabetes vol. 51 S271-S283 (American Diabetes Association Inc., 2002).

67. Carey, A. L. et al. Interleukin-6 increases insulin-stimulated glucose disposal in humans and glucose uptake and fatty acid oxidation in vitro via AMP-activated protein kinase. Diabetes 55, 2688-2697 (2006).

68. Weigert, C. et al. Direct cross-talk of interleukin-6 and insulin signal transduction via insulin receptor substrate-1 in skeletal muscle cells. J. Biol. Chem. 281, 7060-7067 (2006)

69. Łukaszuk, B. et al. A single bout of exercise increases the expression of glucose but not fatty acid transporters in skeletal muscle of IL-6 KO mice. Lipids 47, 763-772 (2012).

70. Glatz, J. F. C., Luiken, J. J. F. P. \& Bonen, A. Membrane fatty acid transporters as regulators of lipid metabolism: Implications for metabolic disease. Physiological Rev. 90, 367-417 (2010).

71. Kiens, B., Kristiansen, S., Jensen, P., Richter, E. A. \& Turcotte, L. P. Membrane associated fatty acid binding protein (FABPpm) in human skeletal muscle is increased by endurance training. Biochem. Biophys. Res. Commun. 231, 463-465 (1997).

72. Greenberg, A. S. et al. Interleukin 6 reduces lipoprotein lipase activity in adipose tissue of mice in vivo and in 3T3-L1 adipocytes: a possible role for interleukin 6 in cancer cachexia. Cancer Res. 52, 4113-4116 (1992).

73. Benrick, A., Wallenius, V. \& Asterholm, I. W. Interleukin-6 mediates exercise-induced increase in insulin sensitivity in mice. Exp. Physiol. 97, 1224-1235 (2012)

74. Serrano, A. L., Baeza-Raja, B., Perdiguero, E., Jardí, M. \& Muñoz-Cánoves, P. Interleukin-6 is an essential regulator of satellite cell-mediated skeletal muscle hypertrophy. Cell Metab. 7, 33-44 (2008).

75. Deng, B., Wehling-Henricks, M., Villalta, S. A., Wang, Y. \& Tidball, J. G. IL-10 triggers changes in macrophage phenotype that promote muscle growth and regeneration. J. Immunol. 189, 3669-3680 (2012).

76. Roth, S. M. et al. Interleukin-6 (IL6) genotype is associated with fat-free mass in men but not women. J. Gerontol. Ser. A Biol. Sci. Med. Sci. 58, B1085-B1088 (2003)

77. Wojewoda, M. et al. Skeletal muscle response to endurance training in IL- $6^{-1-}$ mice. Int. J. Sports Med. 36, 1163-1169 (2015).

78. Adser, $H$. et al. Interleukin-6 modifies mRNA expression in mouse skeletal muscle. Acta Physiologica 202, 165-173 (2011).

79. Li, L. et al. Mitochondrial biogenesis and PGC- $1 \alpha$ deacetylation by chronic treadmill exercise: differential response in cardiac and skeletal muscle. Basic Res. Cardiol. 106, 1221-1234 (2011).

80. Starkie, R., Ostrowski, S. R., Jauffred, S., Febbraio, M. \& Pedersen, B. K. Exercise and IL- 6 infusion inhibit endotoxin-induced TNF- $\alpha$ production in humans. FASEB J. 17, 1-10 (2003).

81. Schindler, R. et al. Correlations and interactions in the production of interleukin-6 (IL- 6), IL-1, and tumor necrosis factor (TNF) in human blood mononuclear cells: IL-6 suppresses IL-1 and TNF. Blood 75, 40-47 (1990).

82. Dagdeviren, S. et al. IL-10 prevents aging-associated inflammation and insulin resistance in skeletal muscle. FASEB J. 31, 701-710 (2017).

83. Troiano, R. P. et al. Physical activity in the United States measured by accelerometer. Med. Sci. Sports Exerc. 40, 181-188 (2008). 
84. Hurst, S. M. et al. IL-6 and its soluble receptor orchestrate a temporal switch in the pattern of leukocyte recruitment seen during acute inflammation. Immunity 14, 705-714 (2001).

85. Ogura, H. et al. Interleukin-17 promotes autoimmunity by triggering a positive-feedback loop via interleukin-6 induction. Immunity 29, 628-636 (2008).

86. Murakami, M. et al. Disease-association analysis of an inflammation-related feedback loop. Cell Rep. 3, 946-959 (2013).

87. Kimura, A. \& Kishimoto, T. IL-6: regulator of $\mathrm{T}_{\mathrm{reg}} / \mathrm{T}_{\mathrm{H}} 17$ balance. Eur. J. Immunol. 40, 1830-1835 (2010).

88. Chiba, Y. et al. Regulation of myelopoiesis by proinflammatory cytokines in infectious diseases. Cell. Mol. Life Sci. 75, 1363-1376 (2018).

89. Hashizume, M., Higuchi, Y., Uchiyama, Y. \& Mihara, M. IL-6 plays an essential role in neutrophilia under inflammation. Cytokine 54, 92-99 (2011).

90. Hotamisligil, G. S. Inflammation and metabolic disorders. Nature $\mathbf{4 4 4}$ 860-867 (2006).

91. Tournadre, A. et al. Changes in body composition and metabolic profile during interleukin 6 inhibition in rheumatoid arthritis. J. Cachexia Sarcopenia Muscle 8, 639-646 (2017).

92. Patsalos, O., Dalton, B. \& Himmerich, H. Effects of IL-6 signaling pathway inhibition on weight and BMI: a systematic review and meta-analysis. Int. J. Mol. Sci. 21, 1-13 (2020).

93. Toussirot, E. et al. Increased high molecular weight adiponectin and lean mass during tocilizumab treatment in patients with rheumatoid arthritis: a 12-month multicentre study. Arthritis Res. Ther. 22, 1-13 (2020).

94. Smolen, J. S. et al. Effect of interleukin- 6 receptor inhibition with tocilizumab in patients with rheumatoid arthritis (OPTION study): a double-blind, placebo-controlled, randomised trial. Lancet 371, 987-997 (2008).

95. Robertson, J. et al. Interleukin-6 blockade raises LDL via reduced catabolism rather than via increased synthesis: a cytokine-specific mechanism for cholesterol changes in rheumatoid arthritis. Ann. Rheum. Dis. 76, 1949-1952 (2017).

96. Otsuka, Y. et al. Effects of tumor necrosis factor inhibitors and tocilizumab on the glycosylated hemoglobin levels in patients with rheumatoid arthritis; an observational study. PLoS ONE 13, e0196368 (2018).

97. Wallenius, V. et al. Interleukin-6-deficient mice develop mature-onset obesity. Nat. Med. 8, 75-79 (2002).

98. Wensveen, F. M., Valentić, S., Šestan, M., Wensveen, T. T. \& Polić, B. The "Big Bang" in obese fat: events initiating obesity-induced adipose tissue inflammation. Eur. J. Immunol. 45, 2446-2456 (2015).

99. Lazar, M. A. How obesity causes diabetes: not a tall tale. Science 307, 373-375 (2005).

100. $\mathrm{Xu}, \mathrm{E}$. et al. Temporal and tissue-specific requirements for T-lymphocyte IL-6 signalling in obesity-associated inflammation and insulin resistance. Nat. Commun. 8, 1-16 (2017).

101. Han, M. S. et al. Regulation of adipose tissue inflammation by interleukin 6. Proc. Natl Acad. Sci. USA 117, 2751-2760 (2020).

102. Mauer, J. et al. Signaling by IL-6 promotes alternative activation of macrophages to limit endotoxemia and obesity-associated resistance to insulin. Nat. Immunol. 15, 423-430 (2014).

103. Whitham, M. et al. Adipocyte-specific deletion of IL-6 does not attenuate obesity-induced weight gain or glucose intolerance in mice. Am. J. Physiol. Endocrinol. Metab. 317, E597-E604 (2019).

104. Wueest, S. et al. Il-6 receptor blockade increases circulating adiponectin levels in people with obesity: an explanatory analysis. Metabolites 11, 1-7 (2021).

105. Zhang, J. et al. Serum interleukin-6 is an indicator for severity in 901 patients with SARS-CoV-2 infection: a cohort study. J. Transl. Med. 18, 1-8 (2020)

106. Chen, L. Y. C., Hoiland, R. L., Stukas, S., Wellington, C. L. \& Sekhon, M. S. Confronting the controversy: interleukin- 6 and the COVID-19 cytokine storm syndrome. Eur. Respir. J. 56, 2003006 (2020).

107. Luporini, R. L. et al. IL-6 and IL-10 are associated with disease severity and higher comorbidity in adults with COVID-19. Cytokine 143, 55507 (2021)

108. WHO Rapid Evidence Appraisal for COVID-19 Therapies (REACT) Working Group et al. Association between administration of IL-6 antagonists and mortality among patients hospitalized for COVID-19: a meta-analysis. JAMA 326, 499-518 (2021).

109. Ascierto, P. A., Fu, B. \& Wei, H. IL-6 modulation for COVID-19: the right patients at the right time? J. Immunother. Cancer 9, e002285 (2021).

110. Sapolsky, R. M., Romero, L. M. \& Munck, A. U. How do glucocorticoids influence stress responses? integrating permissive, suppressive, stimulatory, and preparative actions*. Endocr. Rev. 21, 55-89 (2000).

111. Nadeau., L. \& Aguer, C. Interleukin-15 as a myokine: mechanistic insight into its effect on skeletal muscle metabolism. Appl. Physiol. Nutr. Metab. 44, 229-238 (2019).
112. Knudsen, N. H. et al. Interleukin-13 drives metabolic conditioning of muscle to endurance exercise. Science 368, eaat3987 (2020).

113. Fritsche, L. et al. IL-6 deficiency in mice neither impairs induction of metabolic genes in the liver nor affects blood glucose levels during fasting and moderately intense exercise. Diabetologia 53, 1732-1742 (2010).

114. O'Neill, H. M. et al. IL-6 is not essential for exercise-induced increases in glucose uptake. J. Appl. Physiol. 114, 1151-1157 (2013).

115. Bertholdt, L., Gudiksen, A., Schwartz, C. L., Knudsen, J. G. \& Pilegaard, H. Lack of skeletal muscle IL-6 influences hepatic glucose metabolism in mice during prolonged exercise. Am. J. Physiol. $\mathbf{3 1 2}$ R626-R636 (2017).

116. Gudiksen, A. et al. Lack of skeletal muscle il-6 affects pyruvate dehydrogenase activity at rest and during prolonged exercise. PLoS ONE 11, 1-17 (2016).

117. Molinero, A. et al. Role of muscle IL-6 in gender-specific metabolism in mice. PLoS ONE 12, 1-20 (2017)

118. Senn, J. J. et al. Suppressor of cytokine signaling-3 (SOCS-3), a potential mediator of interleukin-6-dependent insulin resistance in hepatocytes. J. Biol. Chem. 278, 13740-13746 (2003).

119. Bramble, D. M. \& Lieberman, D. E. Endurance running and the evolution of Homo. Nature 432, 345-352 (2004).

120. Kim, H. J. et al. Differential effects of interleukin-6 and -10 on skeletal muscle and liver insulin action in vivo. Diabetes 53, 1060-1067 (2004).

121. Glund, S. et al. Interleukin-6 directly increases glucose metabolism in resting human skeletal muscle. Diabetes 56, 1630-1637 (2007).

122. Steensberg, A., Fischer, C. P., Keller, C., Møller, K. \& Pedersen, B. K. IL-6 enhances plasma IL-1ra, IL-10, and cortisol in humans. Am. J. Physiol. Endocrinol. Metab. 285, 433-437 (2003).

123. Nemet, D., Eliakim, A., Zaldivar, F. \& Cooper, D. M. Effect of rhIL-6 infusion on GH $\rightarrow$ IGF-I axis mediators in humans. Am. J. Physiol. Regulatory Integr. Comp. Physiol. 291, R1663-R1668 (2006).

124. Steeve, K. T., Marc, P., Sandrine, T., Dominique, H. \& Yannick, F. IL-6, RANKL, TNF-alpha/IL-1: interrelations in bone resorption pathophysiology. Cytokine Growth Factor Rev. 15, 49-60 (2004).

125. Fielding, C. A. et al. IL-6 regulates neutrophil trafficking during acute inflammation via STAT3. J. Immunol. 181, 2189-2195 (2008).

126. Diehl, S. \& Rincón, M. The two faces of IL- 6 on $\mathrm{T}_{\mathrm{H}} 1 / \mathrm{T}_{\mathrm{H}} 2$ differentiation. Mol. Immunol. 39, 531-536 (2002).

127. Dominitzki, S. et al. Cutting edge: Trans- signaling via the soluble IL-6R abrogates the induction of FoxP3 in naive CD4 ${ }^{+} \mathrm{CD} 25^{-} \mathrm{T}$ cells. J. Immunol. 179, 2041-2045 (2007).

128. Bettelli, E., Korn, T., Oukka, M. \& Kuchroo, V. K. Induction and effector functions of $\mathrm{T}_{\mathrm{H}} 17$ cells. Nature 453, 1051-1057 (2008).

129. Bode, J. G., Albrecht, U., Häussinger, D., Heinrich, P. C. \& Schaper, F. Hepatic acute phase proteins - regulation by IL-6- and IL-1-type cytokines involving STAT3 and its crosstalk with NF-кB-dependent signaling. Eur. J. Cell Biol. 91, 496-505 (2012).

130. Eskilsson, A. et al. Immune-induced fever is mediated by IL-6 receptors on brain endothelial cells coupled to STAT3-dependent induction of brain endothelial prostaglandin synthesis. J. Neurosci. 34, 15957-15961 (2014).

131. Fernando, M. R., Reyes, J. L., Iannuzzi, J., Leung, G. \& McKay, D. M. The pro-inflammatory cytokine, interleukin-6, enhances the polarization of alternatively activated macrophages. PLoS ONE 9, e94188 (2014).

132. Zhao, J. L. et al. Conversion of danger signals into cytokine signals by hematopoietic stem and progenitor cells for regulation of stress-induced hematopoiesis. Cell Stem Cell 14, 445-459 (2014)

133. Tie, R. et al. Interleukin-6 signaling regulates hematopoietic stem cell emergence. Exp. Mol. Med. 51, 1-12 (2019).

134. Schmidt-Arras, D. \& Rose-John, S. IL-6 pathway in the liver: from physiopathology to therapy. J. Hepatol. 64, 1403-1415 (2016).

135. Kuhn, K. A., Manieri, N. A., Liu, T. C. \& Stappenbeck, T. S. IL-6 stimulates intestinal epithelial proliferation and repair after injury. PLoS ONE 9, e114195 (2014).

136. McLoughlin, R. M. et al. IL-6 trans-signaling via STAT3 directs T cell infiltration in acute inflammation. Proc. Natl Acad. Sci. USA 102 9589-9594 (2005).

137. Narsale, A. A. \& Carson, J. A. Role of interleukin-6 in cachexia: therapeutic Implications. Curr. Opin. Supportive Palliat. Care 8, 321-327 (2014).

138. Johnson, R. W. et al. Glycoprotein 130 (Gp130)/interleukin-6 (IL-6) signalling in osteoclasts promotes bone formation in periosteal and trabecular bone. Bone 81, 343-351 (2015).

139. Pedersen, B. K. \& Febbraio, M. A. Muscle as an Endocrine Organ: Focus on Muscle-derived Interleukin-6. Physiological Rev. 88, 1379-1406 (2008)

140. Wueest, S. \& Konrad, D. The controversial role of IL-6 in adipose tissue on obesity-induced dysregulation of glucose metabolism. Am. J. Physiol. Endocrinol. Metab. 319, E607-E613 (2020).

141. Reilly, S. M. et al. A subcutaneous adipose tissue-liver signalling axis controls hepatic gluconeogenesis. Nat. Commun. 6, 6047 (2015). 
142. Cero, C. et al. $\beta 3$-Adrenergic receptors regulate human brown/beige adipocyte lipolysis and thermogenesis. JCI Insight 6, e139160 (2021).

143. Mohamed-Ali, V. et al. $\beta$-adrenergic regulation of IL-6 release from adipose tissue: in vivo and in vitro studies. J. Clin. Endocrinol. Metab. 86, 5864-5869 (2001).

144. Lyngsø, D., Simonsen, L. \& Bülow, J. Interleukin-6 production in human subcutaneous abdominal adipose tissue: the effect of exercise. J. Physiol. 543, 373-378 (2002).

145. LaPensee, C. R., Hugo, E. R. \& Ben-Jonathan, N. Insulin stimulates interleukin-6 expression and release in LS14 human adipocytes through multiple signaling pathways. Endocrinology 149, 5415-5422 (2008).

146. Kumari, N., Dwarakanath, B. S., Das, A. \& Bhatt, A. N. Role of interleukin-6 in cancer progression and therapeutic resistance. Tumor Biol. 37, 11553-11572 (2016).

147. Puschel, F. et al. Starvation and antimetabolic therapy promote cytokine release and recruitment of immune cells. Proc. Natl Acad. Sci. USA 117, 9932-9941 (2020).

148. Incio, J. et al. Obesity promotes resistance to anti-VEGF therapy in breast cancer by up-regulating IL-6 and potentially FGF-2. Sci. Transl. Med. 10, 945 (2018)

\section{Author contributions}

T. M. K. and D. E. L. designed the study; T. M. K. primarily wrote the original draft with contributions from D. E. L; T. M. K., D. E. L. and B. K. P. reviewed and edited the manuscript.

\section{Competing interests}

All authors declare no competing interests.

\section{Additional information}

\section{Correspondence should be addressed to}

Timothy M. Kistner, Bente K. Pedersen or Daniel E. Lieberman.

Peer review information Nature Metabolism thanks Christoph Garbers, Alan Saltiel and the other, anonymous, reviewers for their contribution to the peer review of this work. Primary handling editor: Ashley Castellanos-Jankiewicz

Reprints and permissions information is available at www.nature.com/reprints.

Publisher's note Springer Nature remains neutral with regard to jurisdictional claims in published maps and institutional affiliations.

(c) Springer Nature Limited 2022 